\title{
HORARIO LABORAL Y SALUD: CONSECUENCIAS PSICOLÓGICAS DE LOS TURNOS DE TRABAJO
}

\author{
JAVIER FERNÁNDEZ-MONTALVO y EVA PIÑOL
}

Universidad Pública de Navarra

(Aceptado el 31 de octubre de 2000)

\begin{abstract}
En este artículo se presenta una revisión de las consecuencias que los turnos de trabajo tienen sobre la salud. Este tipo de horario afecta, aproximadamente, al $20 \%$ de la población laboral. Las investigaciones llevadas a cabo en los últimos años muestran la presencia de numerosas repercusiones negativas sobre la salud de los trabajadores afectados. Desde una perspectiva psicológica destacan los trastornos del sueño, el síndrome de fatiga crónica, el estrés laboral, la sintomatología depresiva y diversos problemas familiares y sociales. Asimismo, se ha observado un mayor número de problemas laborales (absentismo, menor rendimiento y accidentes de trabajo) en este tipo concreto de población laboral, en comparación con los trabajadores con un horario convencional. Por último, se analizan las variables que influyen en la tolerancia al trabajo por turnos.
\end{abstract}

Palabras clave: Horario laboral, turnos rotativos, consecuencias psicológicas, psicopatología laboral.

\section{Labour time and health: psychological consequences of shift work}

In this paper, the impact of shift work on health is analysed. Shift work affects to $20 \%$ of workers. From a psychological point of view, the prevalent negative consequences are the following ones: sleep disorders, chronic fatigue syndrome, stress, depressive symptoms and family and social problems. Likewise, shift workers show highest rate of labour problems (absenteeism, poor efficiency and work-related accidents) than workers with a conventional labour time. Finally, individual and social determinants of shift work tolerance are commented upon.

Key words: Labour time, Shift work, Psychological consequences, Work psychopathology.

\section{INTRODUCCIÓN}

En la sociedad actual una gran parte del tiempo diario se invierte en el trabajo. De hecho, hoy en día el trabajo no es una mera actividad dirigida a ganar dinero, sino que constituye una parte importante de nuestra propia identidad. La realización de un trabajo es, hoy por hoy, imprescindible para integrarse en el medio sociocultural, para ser aceptado por los demás como un sujeto de pleno derecho y para conquistar la libertad per-

Correspondencia: Departamento de Psicología y Pedagogía, Universidad Pública de Navarra, Campus Arrosadía, 31006 Pamplona, Tfno.: 948169830 , Correo-e: fernandez.montalvo@unavarra.es sonal a través de la independencia económica (Fernández-Montalvo, 1998).

Las condiciones en las que se desempeña un puesto de trabajo influyen de forma significativa en la salud mental del trabajador. La oportunidad de control, la adecuación entre las exigencias del puesto y las capacidades de la persona que lo desempeña, las relaciones interpersonales, el salario y la seguridad física son, entre otros, aspectos importantes que ejercen una gran influencia sobre el bienestar psicológico. El grado en que un empleo reúna estos elementos está fuertemente asociado al grado de satisfacción laboral $y$ de salud mental de los trabajadores (Fernández-Montalvo y Garrido, 1999). 
En este contexto, ha comenzado a surgir un interés creciente por el estudio de las alteraciones psicológicas derivadas de la actividad laboral. Fruto de todo ello, ha surgido la denominada psicopatología laboral (cfr. Brodsky, 1996; Cabral, 1988; Fernández-Montalvo, 1998; FernándezMontalvo y Garrido, 1999). Esta especialidad de la psicopatología se dedica al estudio de los trastornos que se desarrollan como consecuencia directa del desempeño de un trabajo, así como de las alteraciones que surgen debido a la ausencia del mismo. Algunos de estos problemas son sobradamente conocidos. El estrés laboral, por ejemplo, constituye probablemente una de las alteraciones psicológicas más estudiadas en los últimos años. Sin embargo, otros trastornos - la adicción al trabajo o las consecuencias negativas del horario laboral, por ejemplo-son relativamente novedosos.

En lo que se refiere a los horarios de trabajo, el sistema de turnos afecta (incluidas las personas con un trabajo exclusivamente nocturno), aproximadamente, al $20 \%$ de la población laboral (Jamal y Baba, 1997; Peiró, 1992). Se trata de un fenómeno relativamente frecuente en nuestra sociedad, que se ha convertido en una forma de vida en los países industrializados, debido a motivaciones económicas y a la necesidad de proporcionar un servicio de 24 horas en una variedad de ocupaciones. Sin embargo, estos ritmos de trabajo presentan grandes inconvenientes: dormir de día no es lo mismo que dormir de noche, y el organismo no se aviene fácilmente a adaptar sus ritmos biológicos a horarios irregulares o a un tiempo de organización artificial cada vez más exigente (Villalba, López, Gavilanes y Legido, 1989).

Desde una perspectiva biológica, la intensidad de las funciones fisiológicas varía a lo largo del día: los máximos suelen coincidir con la luz diurna y los mínimos con la noche. En general, la fase de mayor actividad conlleva un aumento de la glucemia, de la actividad del tiroides y de la temperatura corporal, mientras que la fase de reposo presenta descensos en estas variables (FernándezMontalvo y Garrido, 1999). Se pueden encontrar muchos ejemplos de variables biologicas sujetas a ritmos circadianos: la temperatura corporal (máxima a las 18 horas y mínima a las 6 horas), la secreción de adrenalina (máxima a las 24 horas y mínima a las 6 horas), la secreción de noradrenalina (máxima a las 15 horas y mínima a las 3 horas) y la presión sanguínea (máxima durante el día y mínima por la noche).

De todo ello se deduce que es aconsejable situar el horario laboral entre la salida y la puesta del sol, con el fin de hacer coincidir la actividad laboral con la actividad fisiológica. Sin embargo, las exigencias de la sociedad actual hacen necesario que se establezcan ciertos servicios - los servicios de salud, por ejemplo- en horarios situados fuera de los tiempos fisiologicamente aconsejables. Asimismo, no es ajena a ello la competencia económica existente en el mundo de la empresa que, desde una perspectiva de costes y beneficios, obliga a ajustar el horario laboral a límites extremos. Como consecuencia, no todos los empleados trabajan de 8 horas a 15 horas, y de lunes a viernes.

Los trabajos con un sistema de rotación requieren una adaptación constante de un programa de turnos a otro. De esta for$\mathrm{ma}$, el organismo se encuentra inmerso en un proceso continuo de cambio y de adaptación, por lo que es de esperar que se produzcan más problemas que en el caso de otros horarios más convencionales. Además, la actividad laboral suele tener una periodicidad semanal, que coincide con el tiempo estimado que requiere el organismo para adaptarse a un cambio de turno. 
Por ello, en los últimos años se han comenzado a estudiar las posibles repercusiones que este tipo de horario laboral puede ocasionar en los trabajadores. En este texto se analizan, desde una perspectiva psicológica, los principales resultados encontrados en este sentido.

\section{CONSECUENCIAS DEL HORARIO LABORAL}

El estudio de las consecuencias del trabajo rotativo sobre la salud se ha centrado principalmente en el ámbito físico. Desde una perspectiva psicológica -con la excepción de los estudios sobre los trastornos del sueño- son pocas las investigaciones llevadas a cabo. Sin embargo, ello no significa que este tipo de repercusiones psico(pato)lógicas no sean importantes. Los escasos acercamientos existentes muestran una afectación importante en la salud mental de las personas afectadas por este sistema de trabajo: alteraciones del sueño, síndrome de fatiga crónica, estrés, sintomatología depresiva, etc.

\section{Trastornos del sueño}

Las alteraciones del sueño constituyen el principal problema asociado a los trabajadores por turnos. Sin embargo, resulta muy difícil establecer una tasa de prevalencia aproximada sobre la frecuencia de las mismas en este tipo de población laboral. El grado de ajuste al trabajo por turnos depende de un gran número de variables, entre las que se encuentran la duración del turno, el número de turnos de noche en cada rotación, la dirección (hacia delante o hacia atrás) y velocidad de la rotación, etc. (Kecklund y Akerstedt, 1995; Knauth, 1995). Además, tampoco son ajenos los factores individuales (edad o años de trabajo, por ejemplo) y el estilo de vida de cada trabajador, que pueden modificar los efectos de los turnos sobre el sueño (Fernández-Montalvo y Garrido, 1999; Härmä, 1996; Härmä, Tenkanen, Sjöblom, Alikoski y Heinsal$\mathrm{mi}, 1998$ ). Así se explica el amplio rango encontrado en los estudios epidemiológicos sobre esta temática. De hecho, la tasa de prevalencia de alteraciones del sueño oscila del $20 \%$ al $90 \%$ cuando se trata de trabajadores nocturnos, frente al $10 \%$, como media, en el caso de los trabajadores diurnos (horario partido o 2 turnos de trabajo) (Härmä et al., 1998; Khaleque, 1999; Knauth y Rutenfranz, 1981; Knauth et al., 1980).

Los problemas de sueño los padecen especialmente los trabajadores en turno de noche. Este hecho no resulta extraño puesto que este horario fuerza al individuo a dormir cuando el organismo se inclina a estar despierto y, por el contrario, a permanecer alerta cuando el ciclo del organismo está predispuesto al sueño. Por ello, el patrón de sueño se encuentra notablemente afectado (Goodrich y Weaver, 1998).

Desde un punto de vista psicopatológico, el insomnio constituye el principal trastorno asociado al trabajo por turnos $y$, principalmente, al trabajo nocturno. En concreto, el sueño presenta una disminución importante en su duración y una alteración significativa en su calidad (Costa, 1996; Akerstedt, 1990).

Por lo que se refiere a la duración del sueño, se produce una reducción notable del número de horas destinadas a dormir. En el estudio de Khaleque (1999), por ejemplo, con una muestra de 60 trabajadores de una factoría de tabaco en Bangladesh, la duración del sueño era significativamente menor en los trabajadores con turno de noche $(4,22$ horas diarias, como media) en comparación con los del turno de mañana $(7,15$ horas diarias) o con los del turno de tarde $(7,30$ horas diarias). No son ajenas a esta reducción tan 
importante en las horas del sueño las condiciones ambientales desfavorables asociadas a dormir durante el día (ruidos, llantos de los hijos, teléfono, tráfico, aparatos eléctricos del hogar, excesivo calor en verano, etc.) (Costa, 1996; Knauth y Rutenfranz, 1975). Todo ello dificulta el carácter reparador del sueño y, como consecuencia, aumenta la probabilidad de verse afectado por problemas relacionados con la salud a medio y largo plazo.

Desde un punto de vista cualitativo, se observa una interferencia muy marcada en la distribución de las fases de sueño. En concreto, en el sueño diurno durante el turno de noche se produce una reducción del "sueño profundo», cuando éste último es esencial para recuperarse de la fatiga física. Por otra parte, durante el turno de mañana se eliminan las últimas horas de sueño debido a la necesidad de levantarse temprano. Como consecuencia de ello, se produce una disminución significativa del "sueño paradójico», que es esencial para mantener el bienestar psicologico y que se da con una mayor prevalencia durante la segunda parte del período de sueño (Costa, 1996).

Asimismo, en diversos estudios (Härmä et al., 1998; Khaleque, 1999) se pone de manifiesto la dificultad para mantener el sueño de forma continua (el popular "dormir de un tirón»). De hecho, las interrupciones del sueño son mucho más elevadas en los trabajadores a turnos $y$, especialmente, entre los que trabajan durante la noche.

Además del insomnio, el trastorno del ritmo circadiano constituye otra patología que guarda una estrecha relación con los turnos de trabajo (APA, 1994). Este trastorno se caracteriza por un patrón de sueño desestructurado que obedece a una mala sincronización entre el sistema circadiano endógeno de sueño-vigilia del individuo, por una parte, y las exigencias exógenas de espaciamiento y duración del sueño, por otra.
El diagnóstico del trastorno del ritmo circadiano debe reservarse para cuadros clínicos en los que el individuo presenta un malestar clínicamente significativo o un deterioro social o laboral como consecuencia del mismo. La mayor parte de los afectados no solicitan ayuda terapéutica y no tienen síntomas lo bastante intensos como para permitir efectuar el diagnóstico. Los que acuden a consulta suelen hacerlo cuando el cuadro sintomático ha adquirido una intensidad o una persistencia insoportables. Por ejemplo, no es raro que los trabajadores soliciten ayuda terapéutica después de haberse quedado dormidos durante el trabajo o mientras conducían (APA, 1994).

Este trastorno se puede producir por varias razones: sueño retrasado, cambios horarios -el jet lag-, etc. En el caso concreto de los trabajadores con cambios de turno, el ciclo circadiano endógeno de sueño-vigilia es normal. La alteración se produce por el conflicto entre el patrón de sueño-vigilia generado por el sistema circadiano y el nuevo patrón que exige un cambio de turno de trabajo. Los trabajadores sometidos a frecuentes rotaciones son los que tienen más dificultades, ya que fuerzan el sueño y la vigilia en función de un ritmo circadiano aberrante, lo que imposibilita cualquier intento de adaptacion. Estos trabajadores muestran típicamente una menor duración del sueño y una mayor frecuencia de alteraciones en la continuidad del sueño en comparación con los trabajadores de mañana y de tarde. Asimismo, pueden presentar un mayor grado de somnolencia durante el período en que desearían estar despiertos, es decir, en mitad de la noche, tal como exige su horario laboral. Esta desincronización circadiana suele exacerbarse a causa de unas horas de sueño insuficientes, exigencias sociales y familiares, y alteraciones ambientales (por ejemplo, teléfono, ruido del tráfico, etc.) que perturban sus momentos de descanso. 
Desde un punto de vista biológico se han propuesto dos explicaciones de la influencia del trabajo nocturno en las alteraciones del sueño. Por una parte, este tipo de trastornos se pueden deber a una alteración de los ritmos circadianos provocada por la privación de luz (Moneta, Leclerc, Chastang, Dang y Goldberg, 1996). En concreto, la luz tiene un efecto supresor sobre la secreción de melatonina de forma que, en el caso de los trabajadores nocturnos, activa el organismo cuando al trabajador le toca descansar y lo relaja cuando le toca estar activo. Por otra parte, los trastornos del sueño se pueden deber a una depresión inducida por la falta de luz. En este sentido, los trabajadores nocturnos presentan síntomas similares a los observados en los pacientes con trastornos afectivos estacionales (Healy y Waterhouse, 1991). De hecho, los síntomas de los trabajadores nocturnos se reducen mediante sesiones de exposición a la luz durante el trabajo en el turno de noche (Costa, Ghirlanda, Minors y Waterhouse, 1993; Czeisler, Johnson, Duffy, Brown, Ronda y Kronauere, 1990). En el estudio de Czeisler et al. (1990), por ejemplo, los sujetos que trabajaban de noche y que fueron expuestos a luces brillantes durante esas noches de trabajo, pudieron adaptar sus patrones de sueño-vigilia, de modo que estaban alerta por la noche, dormían bien durante el día y la temperatura de su organismo alcanzaba su nivel máximo por la noche.

Desde una perspectiva más psicosocial, se ha hipotetizado que los trastornos del sueño obedecen a la alteración que se produce en el ámbito familiar y social. Debido a que los trabajadores por turnos, y especialmente los nocturnos, son una minoría, se ven forzados a ajustar su horario al establecido por el resto de la sociedad. De esta forma, las actividades cotidianas (realizar cualquier tipo de gestión, jugar con los hijos, asistir a reunio- nes, comer junto con la familia, etc.) suponen, para estos trabajadores, una reducción importante de las horas de sueño (Moneta et al., 1996).

En cualquier caso, la disminución de la calidad del sueño produce un estado de fatiga persistente, lo que da lugar a una pérdida de eficacia en el trabajo $y$, consecuentemente, a un aumento del esfuerzo necesario para el adecuado rendimiento. Debido a que también falla la recuperación durante los períodos de descanso puede ser habitual sufrir una fatiga crónica. De esta forma, la fatiga mental y física aumentan progresivamente, mientras, paralelamente, disminuye la posibilidad de recuperación. El resultado final de todo ello puede ser la inversión del ritmo sueño/vigilia, caracterizada por el insomnio durante la fase de reposo y por la somnolencia durante la de actividad.

\section{Síndrome de fatiga crónica}

La fatiga no es una enfermedad sino una respuesta biológica normal del organismo cuando se ve sometido a un trabajo, físico o psíquico, de una intensidad superior a la que está acostumbrado. En este sentido, la fatiga ejerce una función adaptativa: actúa como mecanismo de alerta y/o de salvaguardia ante situaciones adversas (Fernández-Montalvo y Garrido, 1999). Afortunadamente una persona en raras ocasiones se deja arrastrar en su trabajo hasta el extremo del agotamiento físico completo - se detendrá bastante antes- o hasta la impotencia psicológica total -antes se ausentará del trabajo-. Por ello, puede decirse que la fatiga, tanto física como psicológica, juega un papel de señal de alarma que impide al organismo llegar a una fase extrema. No obstante, la fatiga interfiere con la coordinación y con la capacidad de vigilancia. Por ello, las personas que 
tienen trabajos que exigen menor coordinación y vigilancia soportan mejor los efectos de la fatiga; pero estos componentes son críticos para el desempeño de muchos trabajos.

En los casos más graves se puede desarrollar el denominado síndrome de fatiga crónica. Se trata de un estado grave de agotamiento físico y mental, que genera un sentimiento generalizado de falta de energía para realizar cualquier labor, que resulta muy imposibilitante para el sujeto, que se agudiza ante cualquier mínimo esfuerzo y que no se explica por un diagnóstico biomédico. Es, en realidad, una denominación moderna de la antigua neurastenia.

Habitualmente, el síndrome aparece acompañado por otros síntomas como, por ejemplo, quejas somáticas -dolores musculares, principalmente-, alteraciones del sueño (Morris, Sharpe, Sharpley, Cowen, Hawton y Morris, 1993), dificultades de atención y de concentración, problemas de memoria y trastornos afectivos (Sharpe, Chalder, Palmer y Wessely, 1997), etc. Todo ello produce una alteración muy importante en la vida cotidiana del sujeto y una disminución significativa del rendimiento laboral.

En general, las personas afectadas por este síndrome acuden a consulta tras pasar varios meses, incluso años, con un estado generalizado de fatiga. Son frecuentes los pensamientos relacionados con la posible presencia de un. virus como factor causante de su estado de agotamiento, lo que genera una gran preocupación. Asimismo, estas personas pasan mucho tiempo pensando en qué pueden hacer para solucionar el problema y terminar con la fatiga. En este sentido, es habitual que reduzcan considerablemente el nivel de actividad, por miedo a que aumenten los síntomas (Sharpe et al., 1997).

El medio ambiente en el que se desarrolla la actividad del individuo tiene una gran repercusión en el índice de fatiga que éste llega a sentir. Los ambientes muy sobrecargados -humos, ruidos, hacinamiento, iluminación inadecuada, etc.- favorecen la aparición de la fatiga. En cualquier caso, la fatiga es uno de los principales síntomas asociados con un ajuste deficiente entre trabajador y puesto de trabajo (Fernández-Montalvo y Garrido, 1999).

Los factores psicológicos que influyen en el desarrollo de este síndrome son muchos y muy diversos, y están estrechamente relacionados entre sí. La falta de interés por el trabajo, el peso de la responsabilidad, la frustración, los conflictos íntimos, la monotonía, la propia sensibilidad, los problemas familiares $\mathrm{y}$ sexuales, etc. Cuando una persona se vuelca en el trabajo como forma de compensar sus fracasos afectivos y/o familiares, presenta, a corto plazo, una especial resistencia a la fatiga y una inusual capacidad de rendimiento. Todo ello, sin embargo, aumenta la probabilidad de desarrollar un cuadro de fatiga crónica a medio o largo plazo.

Por lo que se refiere al horario laboral, se ha comenzado a estudiar recientemente la relación que existe entre los turnos de trabajo y el desarrollo de la fatiga crónica (Akinnawo, 1988; Mounstephen y Sharpe, 1997; Srithongchai y Intaranont, 1996). En este sentido, las jornadas laborales excesivamente largas y los turnos que exigen rotaciones rápidas influyen en el desarrollo de la fatiga crónica (Nicholson y D'Auria, 1999). Sin embargo, la ausencia de estudios empíricos hace que sea muy difícil realizar una valoración minuciosa en este ámbito. Además, el resultado final de fatiga crónica puede deberse tanto a la influencia directa de los turnos de trabajo, como a la ausencia continua de un sueño adecuado y reparador (Rotenberg, Moreno, Benedito y Menna, 1998). 


\section{Estrés laboral}

Se considera que una persona está sometida a una situación de estrés cuando ha de hacer frente a demandas ambientales que sobrepasan sus recursos -o al menos esa es su percepción-, de manera que el sujeto percibe que no puede darles una respuesta efectiva. En este tipo de situaciones, el organismo emite una respuesta de estrés, que consiste en un importante aumento de la activación fisiológica y cognitiva del organismo, que, a su vez, se prepara para una intensa actividad motora. Las consecuencias de esta activación dependen de su duración e intensidad. Así, cuando la respuesta de estrés es demasiado frecuente, intensa o duradera puede tener repercusiones negativas, con una amplia gama de manifestaciones orgánicas: los llamados trastornos psicofisiológicos. En cualquier caso, la respuesta de estrés depende en parte de las demandas de la situación, y en parte de las habilidades, recursos o forma de comportarse de la persona en dicha situación (Labrador y Crespo, 1993).

Desde una perspectiva laboral, son varios los factores claramente identificados como estresores (Peir6, 1992): a) el ambiente físico, b) las demandas del trabajo, c) los contenidos del trabajo, d) el desempeño de roles, las relaciones interpersonales y el desarrollo de la carrera, e) las nuevas tecnologías, y f) las fuentes extraorganizacionales. En concreto, los turnos rotativos forman parte de las demandas estresantes del trabajo.

En este sentido, existe una estrecha relación entre el trabajo en un sistema de turnos rotativos y el estrés laboral (Costa, 1996; Nicholson y D'Auria, 1999). El constante proceso de adaptación al que se ven sometidos este tipo de trabajadores supone una fuente constante de estrés. A medida que transcurre el tiempo, la continua activación del organismo trae como consecuencia problemas en la salud. De hecho, los trastornos físicos más frecuentes en las personas que trabajan en turnos rotativos son los gastrointestinales y los cardiovasculares.

No obstante, en otros casos no se han confirmado estos datos. Por poner un ejemplo, en el estudio de Jamal y Baba (1997), con una muestra de 175 enfermeras de un hospital de Canadá, no se encontraron diferencias significativas entre los 4 tipos de turnos estudiados (fijo día, fijo tarde, fijo noche y rotativo) con respecto al estrés. Todo ello acentúa aún más la necesidad de fomentar las investigaciones relacionadas con los turnos laborales y sus consecuencias negativas en la salud.

\section{Sintomatología depresiva}

Son pocos los estudios que han examinado la influencia del trabajo por turnos sobre el estado de ánimo. Sin embargo, la combinación de todos los problemas encontrados en este tipo de trabajadores aumenta considerablemente la probabilidad de desarrollar sintomatología depresiva (Goodrich y Weaver, 1998). En el estudio de Alfredsson, Akerstedt, Mattsson y Wilborg (1991), por ejemplo, se encontró un nivel significativamente más elevado de depresión entre los guardias de seguridad con turno permanente de noche que entre los que trabajaban con un horario tradicional. Unos resultados similares se han obtenido también en otros estudios con trabajadores a turnos (Bohle y Tilley, 1989), pero no se han confirmado en otros (Goodrich y Weaver, 1998).

En cualquier caso, existe una conexión entre la pobreza de sueño y el desarrollo de síntomas depresivos. En concreto, en el estudio de Ford y Kamerow (1989) las personas que sufrían de insomnio presentaban un mayor riesgo de desarrollar un 
trastorno depresivo que los sujetos con un sueño normal. Además, estos mismos autores observaron cómo disminuía el riesgo de depresión cuando se trataba el insomnio. Por ello, una posible explicación de la mayor probabilidad de desarrollar una depresión en este tipo de trabajadores podría radicar en la alta prevalencia del insomnio entre los mismos.

Por lo tanto, se necesita un mayor número de investigaciones sobre este tema y aclarar así los resultados contradictorios obtenidos en algunos estudios. Además, desde el punto de vista etiológico, hoy por hoy no está lo suficientemente clara la vía de influencia del sistema de turnos en el desarrollo de la sintomatología depresiva. ¿Es el propio sistema de turnos el que influye en el estado de ánimo? o, como parece más probable, ¿son las deficiencias del sueño las que producen una mayor presencia de la sintomatología depresiva entre los trabajadores a turnos? Si esto es así, las alteraciones del sueño se configuran como la repercusión principal de este tipo de horario laboral, que provoca directa o indirectamente la mayor parte de las consecuencias observadas en los trabajadores.

\section{Problemas psicosociales}

Desde un punto de vista psicosocial, el trabajo por turnos, y especialmente el nocturno, provoca una alteración en el ámbito familiar y social. Estos trabajadores constituyen una minoría y, por lo tanto, se ven forzados a ajustar su horario al del resto de la sociedad. Todo ello, puede ser una fuente importante de conflictos (problemas en la relación de pareja, escaso contacto con los hijos, incompatibilidad de horarios con los amigos o problemas para disfrutar del tiempo libre, entre otros). Se produce, en definitiva, una disminución de la calidad de vida (Puca, Perrucci, Prudenzano, Savarese,
Misceo, Perilli, Palumbo, Libro y Genco, 1996).

En el estudio de Khaleque (1999), los resultados obtenidos mostraron que el $\mathbf{9 0 \%}$ de los trabajadores de la muestra presentaba alteraciones importantes en la vida familiar. Asimismo, un $87 \%$ informaba de restricciones significativas en las relaciones sociales, un $91 \%$ de una disminución de las actividades de ocio y un $85 \%$ de irregularidades en el horario de comida familiar. En otra investigación anterior de este mismo grupo (Khaleque y Wadud, 1996), las conclusiones iban en la misma línea; pero en aquella ocasión, cuando se compararon los hombres y las mujeres, se observó que ellas se veían aún más afectadas.

En cualquier caso, todas estas dificultades para mantener las relaciones sociales y familiares habituales provocan, a su vez, efectos negativos en la relación de pareja y en el cuidado de los hijos (Costa, 1996). La ausencia de un horario familiar común, en el que coincidan las horas dedicadas al descanso y a los momentos de ocio, impide, en muchas ocasiones, el establecimiento de una programación familiar adecuada. Asimismo, por lo que se refiere a la relación de pareja, puede ser relativamente habitual la disminución de la frecuencia de las relaciones sexuales: la «mañana» de uno es la "noche» del otro, el "descanso" de uno es el "cansancio" del otro.

Sin embargo, el trabajo rotativo no solamente influye en el propio trabajador afectado. De hecho, se ha encontrado un impacto emocional importante en los hijos de los trabajadores a turnos. En concreto, en el estudio de Barton, Aldridge y Smith (1998), las hijas, pero no los hijos varones, presentaban una sintomatología depresiva significativamente mayor en comparación con las hijas de trabajadores con un horario exclusivamente diurno. Además, la percepción de sus habilidades escolares era también significativamente peor. 


\section{PROBLEMAS LABORALES ESPECÍFICOS}

En el ámbito de la empresa, existen tres indicadores básicos que reflejan el grado de adaptación del trabajador a su puesto profesional: el rendimiento, el absentismo y los accidentes de trabajo. La alteración en uno o más de estos tres factores supone, habitualmente, la existencia de alguna dificultad por parte del trabajador y, más en concreto, de problemas relacionados con la salud. Así, por ejemplo, la implicación en una conducta adictiva provoca una disminución del rendimiento, un mayor índice de absentismo y un mayor número de accidentes de trabajo (Fernández-Montalvo, Báez y Echeburúa, 2000; FernándezMontalvo y Echeburúa, 2000). Lo mismo ocurre con el estrés laboral (Peiro, 1992) o con la presencia de un trastorno del estado de ánimo (Pacheco, Jiménez e Iglesia, 1990).

Por ello, se han aplicado también estos tres parámetros al estudio de las consecuencias de los turnos de trabajo. Por lo que se refiere al rendimiento, las mismas tareas realizadas en turnos diurnos y nocturnos pueden alcanzar diferencias productivas de hasta un $15 \%$ (Martín, Martínez-Arias y Pereda, 1989). Estas diferencias se han relacionado con las variaciones de las funciones fisiologicas en función de los ritmos circadianos, así como con los cronotipos matutino y vespertino que se explicarán en el siguiente apartado (Martín, Díaz y Rubio, 1995).

Desde la perspectiva del absentismo, los estudios muestran resultados contradictorios: algunos señalan la existencia de más absentismo laboral entre los trabajadores diurnos; otros entre los trabajadores a turnos; y otros no encuentran diferencias entre ambos (Costa, 1996; Jamal y Baba, 1997). No obstante, a pesar de esta incoherencia en los resultados obtenidos, está bien documentada la mayor presencia de problemas relacionados con la salud física -alteraciones cardiovasculares y gastrointestinales, principalmente- entre los trabajadores a turnos (Costa, 1996; Nicholson y D'Auria, 1999; Tucker, Smith, Macdonald y Folkard, 1998; Villalba et al., 1989). Como consecuencia de ello, cabe esperar un mayor absentismo entre este tipo de población laboral. Sin embargo, con los datos actuales, resulta muy difícil valorar adecuadamente la incidencia concreta de los turnos laborales en el nivel de absentismo.

Por último, en relación con los accidentes de trabajo, los ritmos circadianos provocan una disminución de la ejecución psicofísica durante la noche que, junto con las deficiencias de sueño y los fuertes sentimientos de fatiga, disminuyen la eficiencia en el trabajo y aumentan la posibilidad de errores y de accidentes. Sin embargo, los estudios acerca de los accidentes laborales entre los trabajadores a turnos son muy controvertidos, sin que exista una conclusión clara de la relación entre ambas variables. En cualquier caso, no se debe olvidar que dos accidentes nucleares recientes (Three Mile Island, 1979 y Chernobyl, 1984) comenzaron en las horas nocturnas ( $4 \mathrm{~h}$. y $1,25 \mathrm{~h}$. respectivamente).

\section{TOLERANCIA A LOS TURNOS LABORALES}

El trabajo en un sistema de turnos influye negativamente en la salud. Sin embargo, no todas las personas expuestas a un mismo sistema de turnos responden igual. El grado de tolerancia a los turnos de trabajo varía considerablemente en función de variables individuales, familiares, sociales, laborales y, lógicamente, de variables relacionadas con el propio sistema de turnos (Tabla 1). 
Tabla 1. Factores que influyen en la tolerancia al trabajo por turnos (Costa, 1996, modificado)

Características individuales

Edad. Sexo. Ejercicio físico. Años de trabajo en un sistema de turnos. Rasgos de personalidad (introversión-extraversión, neuroticismo). Estructura circadiana. Cronotipos (matutinidad-vespertinidad). Hábitos de alimentación y de sueño.

Características familiares

Estado marital. Número y edad de los hijos. Nivel socioeconómico. Trabajo por turnos de la pareja. Actitudes familiares.

\section{Características sociales}

Mercado laboral. Tradición del trabajo por turnos en la comunidad. Actividades de ocio. Apoyo social. Actitudes de la comunidad.

\section{Caracteristicas laborales}

Sector laboral. Horas de trabajo. Ambiente de trabajo. Características del puesto desempeñado. Nivel de ingresos. Cualificación. Satisfacción con el trabajo. Oportunidad para el desarrollo de la carrera. Relaciones humanas.

Características de los turnos

Turnos continuos o semicontinuos. Turnos rotativos o permanentes. Dirección de la rotación (hacia delante o hacia atrás). Duración del ciclo de turnos. Número de noches consecutivas. Número de noches por año. Fines de semana libres por cada ciclo de turnos. Horario del comienzo y del final del turno.

Desde una perspectiva individual, algunos estudios señalan que los trabajadores de mayor edad y las mujeres son las que sufren con más frecuencia las repercusiones negativas debidas a los turnos laborales (Marquie y Foret, 1999; Marquie, Foret y Queinnec, 1999). De hecho, en el caso concreto de las mujeres se ha encontrado una mayor frecuencia de alteraciones del ciclo menstrual, de abortos y de partos prematuros, así como menores tasas de embarazos (Axelson, Rylander y Molin, 1989; McDonald, McDonald, Armstrong, Cherry, Nolin y Robert, 1988; Nurminen, 1998; Uehata y Sasakawa, 1982).

En relación con las variables de personalidad son muy pocos los estudios llevados a cabo, por lo que se carece de conclusiones sólidas. No obstante, parece que las personas con un alto grado de introversión y de neuroticismo tienen mayores repercusiones negativas cuando trabajan en un sistema de turnos (Colquhoun y Folkard, 1978).

Por otra parte, también se ha estudiado la relación existente entre los cronotipos más extremos (matutinos y vespertinos) y la tolerancia al trabajo por turnos. Ambos presentan un rendimiento laboral diferente en función del horario de trabajo (Hilderbrant y Stratmann, 1979; Khaleque, 1999; Tankova, Sierra, Álvarez-Castro y Buela-Casal, 1996).

El cronotipo matutino se refiere a aquellas personas que presentan un rendimiento sensiblemente mejor en los primeros momentos del día (por la mañana) y peor en los últimos (al atardecer). Habitualmente, muestran una preferencia por irse temprano a la cama y levantarse pronto por la mañana. En el otro extremo del continuo se encuentra el cronotipo vespertino. Este hace referencia a aque- 
llas personas que realizan una mejor ejecución de las tareas en los últimos momentos del día y peor en los primeros. Este tipo de sujetos prefiere irse tarde a la cama y, en consecuencia, levantarse también tarde al día siguiente.

Todo ello lleva a que los tipos matutinos tengan mayores dificultades a la hora de afrontar el turno de noche, mientras que a los tipos vespertinos les cuesta mucho más el turno de mañana (Akerstedt y Torsvall, 1981). En esta misma línea, son varios los estudios que señalan la existencia de un mayor número de consecuencias negativas entre las personas con un cronotipo matutino sometidas a un turno rotativo con ciclo nocturno (Akerstedt, 1988; Khaleque, 1999; Hilderbrant y Stratmann, 1979; Martín et al., 1995). Por ello, se ha hipotetizado que los pertenecientes al tipo vespertino se adaptarían mejor al trabajo por turnos rotativos. No obstante, es necesario un mayor número de investigaciones sobre este tema.

Desde una perspectiva familiar, hay también una serie de variables que dificultan la adaptación al trabajo por turnos. Entre los principales factores encontrados destacan, entre otros, la ausencia de una pareja que apoye al trabajador (Beermann y Nachreiner, 1995; Loudon y Bohle, 1997) y el hecho de tener hijos, con el aumento asociado de las obligaciones domésticas (Loudon y Bohle, 1997; Rotenberg, Portela, Moreno, Benedito-Silva y Menna, 1997).

Por último, en relación con las características de los turnos, cada vez hay una mayor evidencia empírica que demuestra que no todos los sistemas de rotación provocan las mismas consecuencias sobre el sueño de las personas afectadas. En este sentido, un tipo de horario que parece especialmente negativo para la salud es el que plantea cambios de turno breves (2-3 días) y frecuentes de diario a nocturno Este sistema exige un estado de continua adaptación del organismo al horario de trabajo. Algunos autores señalan incluso que lo más adecuado sería mantener durante espacios de tres semanas el mismo horario laboral (Fernández-Montalvo y Garrido, 1999; Jamal y Jamal, 1982).

Asimismo, diversos estudios muestran que la rotación hacia atrás (del turno de mañana al de noche y de éste al de tarde) conlleva más dificultades de adaptación que la rotación hacia delante (del turno de mañana al de tarde y de éste al de noche) (Jamal y Jamal, 1982; Khaleque, 1999; Knauth, 1993, 1995; Monk, 1990). Esta última provoca un mejor ajuste circadiano de los trabajadores y una disminución de las consecuencias sobre la salud

En cualquier caso, el estudio de las variables que influyen en el grado de adaptación al trabajo por turnos resulta fundamental para disminuir los riesgos observados en esta población laboral. Una revisión más amplia en este sentido puede encontrarse en Nachreiner (1998).

\section{CONCLUSIONES}

Las investigaciones que se han llevado a cabo en los últimos años muestran que el trabajo en un sistema de turnos rotativos ocasiona numerosos problemas en la salud de las personas afectadas. De hecho, se estima que aproximadamente el $20 \%$ de los trabajadores tienen que abandonar el trabajo por turnos en un corto espacio de tiempo debido a serios problemas de salud (Costa, 1996). Este sistema de trabajo actúa, por lo tanto, como un factor de riesgo que favorece el desarrollo de determinadas alteraciones, después de una exposición prolongada al mismo. Además, el horario laboral puede actuar como un factor estresante adicional, no sólo debido a la desincronización entre los ritmos biológicos endógenos y los ritmos externos, sino también debido al sueño insuficiente y a las dificultades familiares y sociales. Todas estos proble- 
mas se ven notablemente aumentados en el caso concreto de los trabajadores nocturnos.

Desde una perspectiva psicológica, las alteraciones del sueño se perfilan como el principal problema asociado a este sistema de trabajo. En concreto, la necesidad constante de rotar por los diversos turnos establecidos influye directamente sobre la cantidad y calidad del sueño. Como consecuencia, se altera la principal función del sueño: la función reparadora. La ausencia de un sueño adecuado aumenta la probabilidad de padecer otro tipo de trastornos: aumento de la ansiedad, alteraciones del estado de ánimo, estrés, fatiga crónica, etc. Además, estos patrones de sueño equivocados repercuten directamente sobre la actividad laboral: el rendimiento de los trabajadores por turnos rotativos es, en general, menor de noche que de día; este tipo de trabajadores tiene una mayor probabilidad de sufrir accidentes laborales; y, por otra parte, las tasas de absentismo laboral aumentan considerablemente (Villaba et al., 1989).

Sin embargo, las consecuencias psicológicas negativas descritas en este texto varían considerablemente de unas personas a otras. Ello refleja la influencia de otro tipo de variables (personales, familiares, sociales, etc.) en el grado de tolerancia a los turnos rotativos. Por este motivo, se ha comenzado a investigar acerca de posibles sistemas de selección específicos para trabajadores rotativos. En este sentido, el conocimiento de la tipología más adaptativa al trabajo por turnos ofrece la posibilidad de establecer los turnos laborales en función de las características individuales de los trabajadores. Para ello, es necesario tener en cuenta las variables fisiológicas, psicologicas, sociales y ambientales, que rodean a cada trabajador (ritmos circadianos, cronotipos, hábitos de sueño y de comida, preferencias personales de turno laboral, papel de la familia, actividades socio- culturales, condiciones de trabajo, etc.). Sin embargo, a pesar de resultar interesantes este tipo de perspectivas, hoy en día se carece de un cuerpo sólido de conocimientos sobre las características más adaptativas para realizar un trabajo rotativo. Por otra parte, tampoco se conoce con exactitud el tipo concreto de turno que genera un menor número de problemas de salud entre los trabajadores.

No obstante, existen una serie de características que desaconsejan el trabajo en un sistema de turnos rotativos $y$, especialmente, en el turno de noche $\mathrm{y}$ que se recogen en la Tabla 2 (Koller, 1996). Todas ellas suponen un claro riesgo para la salud de los trabajadores. De hecho, desde una perspectiva médica, se considera que los trabajadores con un sistema de turnos rotativos constituyen una población de riesgo para el desarrollo de determinadas enfermedades. Por ello, parece necesario que los exámenes de salud sean más frecuentes que los contemplados actualmente en la legislación laboral. Estas revisiones deben contemplar la evaluación de los síntomas más frecuentes entre esta población: gastrointestinales, cardiovasculares y circulatorios; así como la existencia de abuso de drogas y de problemas relacionados con el sueño, así como cualquiera de las alteraciones descritas en este texto. Sólo de esta forma se podrá conseguir una detección precoz de las dificultades de estos trabajadores.

Por último, un aspecto fundamental radica en la posibilidad de prevenir este tipo de alteraciones que tantos problemas psicológicos, sociales y, desde el punto de vista empresarial, económicos provoca en las personas afectadas. Las organizaciones sindicales han mostrado su preocupación en este sentido. La solución propuesta es la eliminación del trabajo nocturno en aquellas tareas que no son «absolutamente necesarias" (Martín et al., 1995). Los resultados obtenidos por diversas inves- 
Tabla 2. Condiciones que desaconsejan el trabajo nocturno (Koller, 1996)

Absolutamente no recomendable si están presentes una o más de las siguientes condiciones:

- Enfermedad gastrointestinal grave (incluye enfermedad hepática o pancreática grave)

- Diabetes, especialmente la insulinodependiente

- Irregularidades hormonales graves

- Epilepsia

- Factores de alto riesgo cardiovascular

- Estados depresivos

- Estados psicóticos

- Trastornos crónicos del sueño

No recomendable debido a una posible agravación de los efectos adversos si están presentes una o más de las siguientes condiciones:

- Más de 45-50 años

- Condiciones domésticas insatisfactorias para dormir

- Ausencia de apoyo social y familiar

- Mujer con niños pequeños

tigaciones (Knauth, 1993; Monk, 1990) apuntan también a la necesidad de reducir al máximo el turno de noche. Sin embargo, el análisis económico de la realidad laboral actual hace difícil la desaparición de los turnos laborales.

Por ello, cobra una especial importancia el desarrollo de programas y de estrategias de preparación, que puedan reducir los efectos adversos de los turnos de trabajo. En algunas empresas se han llevado a cabo programas de este tipo, con un fuerte componente educativo, en los que se instruye a los trabajadores sobre aspectos tan básicos como la dieta más apropiada, el número de horas de sueño recomendables, el período adecuado de aislamiento durante el sueño diurno o las estrategias más eficaces para afrontar los turnos rotativos y, especialmente, el trabajo nocturno, entre otras (Koller, 1996; Martín et al., 1995). Sin embargo, se carece de datos concretos sobre la eficacia de este tipo de programas.

Asimismo, algunos autores (Jamal, 1989; Monk y Folkard, 1992; Wedderburn y Scholarios, 1993) señalan una serie de características que deben contemplarse en los sistemas de turnos, con el objetivo de reducir al máximo las consecuencias negativas sobre la salud: a) aumentar el tiempo del período de rotación, b) establecer una rotación hacia delante (mañana-tarde-noche), c) proporcionar incentivos económicos razonables por el trabajo a turnos, d) establecer la posibilidad de elegir el sistema de turnos, y e) informar correctamente a los trabajadores acerca de su horario de turnos, con el objetivo de poder planificar sus actividades.

En cualquier caso, la gravedad de los problemas encontrados en los trabajadores por turnos exige un aumento importante de las investigaciones dirigidas a este tema concreto. Desgraciadamente, la psicología clínica se ha ocupado muy poco del ámbito de las organizaciones. De hecho, este campo ha estado reservado, casi en exclusiva, a la psicología social. Probablemente el aumento de la demanda clínica motivada por problemas psicológicos derivados del horario laboral, actuará, en los próximos años, como motor de arranque de este tipo de estudios. 


\section{REFERENCIAS BIBLIOGRÁFICAS}

Akerstedt, T. (1988). Sleepiness as a consequence of shift work. Sleep, 11, 17-34.

Akerstedt, T. (1990). Psychological and psychophysiological effects of shiftwork. Scandinavian Journal of Work Environment and Health, 16, 67-73.

Akerstedt, T., y Torsvall, L. (1981). Shift work: shift-dependent well-being and individual differences. Ergonomics, 24,265-273.

Akinnawo, E.O. (1988). Psychopathology of shift work. Social Behavior and Personality, 17, 103-109.

Alfredsson, L., Akerstedt, T., Mattsson, M., y Wilborg, B. (1991). Self-reported health and well-being amongst night security guards: a comparison with the working population. Ergonomics, 34, 525-530.

American Psychiatric Association (1994). Diagnostic and statistical manual of mental disorders ( $4^{\text {th }}$ ed.). Washington, DC: Author.

Axelson, G., Rylander, R., y Molin, I. (1989). Outcome of pregnancy in relation to irregular and inconvenient work schedules. British Journal of Industrial Medicine, 46, 393-398.

Barton, J., Aldridge, J., y Smith, P. (1998). The emotional impact of shift work on the children of shift workers. Scandinavian Journal of Work Environment and Health, 24, 146-150.

Beermann, B., y Nachreiner, F. (1995). Working shifts. Different effects for women and men? Work Stress, 9, 289-297.

Bohle, P., y Tilley, A.J. (1989). The impact of night work on psychological well-being. Ergonomics, 32, 1089-1099.

Brodsky, C.M. (1996). The psychiatry of work. Occupational Medicine, 11, 685-698.

Cabral, C.A. (1988). Psicopatología del trabajo. Acta Psiquiátrica y Psicológica de América Latina, 34, 237-242.

Colquhoun, W.P., y Folkard, S. (1978). Personality differences in body temperature rhythm, and their relation to its adjustment to nighty work. Ergonomics, 21, 811-817.

Costa, G. (1996). The impact of shift and night work on health. Applied Ergonomics, 27, 9-16.
Costa, G., Ghirlanda, G., Minors, D.S., y Waterhouse, J.M. (1993). Effects of bright light on tolerance to night work. Scandinavian Journal of Work Environment and Health, 19, 414-420.

Czeisler, C.A., Johnson, M.P., Duffy, J.F., Brown, E.N., Ronda, J.M., y Kronauere, R.E. (1990). Exposure to bright light and darkness to treat physiologic maladaptation to night work. New England Journal of Medicine, 322, 1253-1259.

Fernández-Montalvo, J. (1998). Los trastornos psicológicos derivados del trabajo: hacia una psicopatología laboral. Clínica $y$ Salud, 9, 607-620.

Fernández-Montalvo, J., y Echeburúa, E. (2000). El consumo excesivo de alcohol: un reto para la salud laboral. Salud y Drogas.

Fernández-Montalvo, J., y Garrido, E. (1999). Psicopatología laboral. Pamplona. Servicio de Publicaciones de la Universidad Pública de Navarra.

Fernández-Montalvo, J., Báez, C., y Echeburúa, E. (2000). Ludopatía y trabajo: análisis de las repercusiones laborales de los jugadores patologicos de máquinas tragaperras. Clínica y Salud, 11, 5-14.

Ford, D.E., y Kamerow, D.B. (1989). Epidemiology study of sleep disturbances and psychiatric disorders. Journal of the American Medical Association, 262, 1479-1484.

Goodrich, S., y Weaver, K.A. (1998). Differences in depressive symptoms between traditional workers and shiftworkers. Psychological Reports, 83, 571-576.

Härmä, M. (1996). Ageing, physical fitness and shift work tolerance. Applied Ergonomics, 27, 25-29.

Härmä, M., Tenkanen, L., Sjöblom, T., Alikoski, T., y Heinsalmi, P. (1998). Combined effects of shift work and life-style on the prevalence of insomnia, sleep deprivation and daytime sleepiness. Scandinavian Journal of Work Environment and Health, 24, 300-307.

Healy, D., y Waterhouse, J.M. (1991). Reactive rhythms and endogenous clocks. Psychological Medicine, 21, 557-564.

Hilderbrant, G., y Stratmann, I. (1979). Circadian system response to night work in relation to the individual circadian phase posi- 
tion. International Archives of Occupational and Envioronmental Health, 43, 73-83. Jamal, M. (1989). Shiftwork creates unusual problems. Personnel Journal, 68, 114-117. Jamal, M., y Baba, V. (1997). Shiftwork, burnout and well-being: a study of Canadian nurses. International Journal of Stress Management, 4, 197-204.

Jamal, M., y Jamal, S.M. (1982). Work and nonwork experiences of employees on fixed and rotating shifts. Journal of Vocational Behavior, 20, 282-293.

Kecklund, G., y Akerstedt, T. (1995). Effects of timing of shifts on sleepiness and sleep duration. Journal of Sleep Research, 4, 3740.

Khaleque, A. (1999). Sleep deficiency and quality of life of shift workers. Social Indicators Research, 46, 181-189.

Khaleque, A., y Wadud, N. (1996). Job attitudes and psychosocial problems of shift and day workers. Journal of the Indian Academy of Applied Psychology, 22, 95-100.

Knauth, P. (1993). The design of shift systems. Ergonomics, 36, 15-28.

Knauth, P. (1995). Speed and direction of shift rotation. Journal of Sleep Research, 4, 41-469.

Knauth, P., y Rutenfranz, J. (1975). The effects of noise on the sleep of nightworkers. En W.P. Colquehourn, S. Folkard, P. Knauth y J. Rutenfranz (Eds.), Experimental studies of shiftwork. Westdeutscher.

Knauth, P., y Rutenfranz, J. (1981). Duration of sleep related to the type of shiftwork. En A. Reinberg, N. Vieux y P. Andlauer (Eds.), Night and shiftwork: Biological and social aspects. Oxford. Pergamon Press.

Knauth, P., Rutenfranz, J., Schulz, H., Bruder, S.I., Romberg, H.P., y Decoster, R. (1980). Experimental shift work studies of permanent night and rapidly rotating shift systems. International Archives of Occupational and Environmental Health, 46, 111-125.

Koller, M. (1996). Occupational health services for shift and night workers. Applied Ergonomics, 27, 31-37.

Labrador, F.J., y Crespo, M. (1993). Estrés. Trastornos psicofisiológicos. Madrid. Eudema.

Loudon, R., y Bohle, P. (1997). Work/non work conflict and health in shiftwork: relationships with family status and social support. International Journal of Occupational and Environmental Health, 3, 71-77.

Marquie, J.C., y Foret, J. (1999). Sleep, age and shiftwork experience. Journal of Sleep Research, 8, 297-304.

Marquie, J.C., Foret, J., y Queinnec, Y. (1999). Effects of age, working hours and job content on sleep: a pilot study. Experimental Aging Research, 25, 421-427.

Martín, J., Díaz, E.M., y Rubio, S. (1995). Hacia un modelo de selección específico para trabajadores en turnos rotativos. Ansiedad y Estrés, 1, 173-187.

Martín, J., Martínez-Arias, M.R., y Pereda, S. (1989). Análisis y predicción del rendimiento laboral en tareas rotativas a partir de indicadores fisiológicos. Revista de Psicología del Trabajo y de las Organizaciones, 5, 14-26.

McDonald, A.D., McDonald, J.C., Armstrong, B., Cherry, N.M., Nolin, A., y Robert, D. (1988). Fetal death and work in pregnancy. British Journal of Industrial Medicine, 45, 148-157.

Moneta, G.B., Leclerc, A., Chastang, J.F., Dang, P., y Goldberg, M. (1996). Time-trend of sleep disorder in relation to night work: a study of sequential 1-year prevalences within the GAZEL cohort. Journal of Clinical Epidemiology, 49, 1133-1141.

Monk, T.H. (1990). The relationship of chronobiology to sleep schedules and performance demands. Work and Stress, 4, 227-236.

Monk, T.H., y Folkard, S. (1992). Making shiftwork tolerable. New York: Taylor \& Franics.

Morris, R., Sharpe, M., Sharpley, A., Cowen, P., Hawton, K., y Morris, J. (1993). Abnormalities of sleep in patients with the chronic fatigue syndrome. British Medical Journal, 306, 1161-1164.

Mounstephen, A.H., y Sharpe, M. (1997). Chronic fatigue syndrome and occupational health. Occupational Medicine, 47, 217-227.

Nicholson, P.J., y D'Auria, D.A. (1999). Shift work, health, the working time regulations and health assessment. Occupational Medicine, 49, 127-137.

Nurminen, T. (1998). Shift work and reproductive health. Scandinavian Journal of Work Environment and Health, 24, 28-34. 
Pacheco, L., Jiménez, P., y De la Iglesia, A. (1990). Estudio médico-descriptivo de la depresión en el ámbito laboral. Salud y Trabajo, 82, 31-36.

Peiró, J.M. (1992). Desencadenantes del estrés laboral. Madrid. Eudema.

Puca, F.M., Perrucci, S., Prudenzano, M.P., Savarese, M.A., Misceo, S., Perilli, S., Palumbo, M., Libro, G., y Genco, S. (1996). Quality of life in shift work syndrome. Functional Neurology: New Trends in Adaptive and Behavioral Disorders, 11, 261-268.

Rotenberg, L., Moreno, C., Benedito, A.A., y Menna, L. (1998). Sleep/wake cycle parameters and sleep/fatigue complaints in female night workers. Biological Rhythm Research, 29, 585-590.

Rotenberg, L., Portela, L.F., Moreno, C., Benedito, A.A., y Menna, L. (1997). Sleep and sleep/fatigue complaints in female nightworkers at an assembly line: differences between those who have and those who do not have young children. Shiftwork International Newslater, 14, 85

Sharpe, M., Chalder, T., Palmer, I., y Wessely, S. (1997). Chronic fatigue syndrome. A practical guide to assessment and management. General Hospital Psychiatry, 19, 185-199.
Srithongchai, S. e Intaranont, K. (1996). A study of impact of shift work on fatigue level of workers in a sanitary-ware factory using a fuzzy set model. Journal of Human Ergology, 25, 93-99.

Tankova, I., Sierra, J.C., Álvarez-Castro, S., y Buela-Casal, G. (1996). Influencia de la matutinidad-vespertinidad sobre el rendimiento y variables psicofisiológicas. Vigilia y Sueño, 8, 35-45.

Tucker, P., Smith, L., Macdonald, I., y Folkard, S. (1998). The impact of early and late shift changeovers on sleep, health, and well-being in 8-and 12-hour shift systems. Journal of Occupational Health Psychology, 3, 265-275.

Uehata, T., y Sasakawa, N. (1982). The fatigue and maternity disturbances of night work women. Journal of Human Ergonomy, 11, 465-474.

Villalba, J.A., López, J., Gavilanes, M., y Legido, J.C. (1989). Repercusión fisiológica del trabajo nocturno sobre la salud. Salud y Trabajo, 75, 17-20.

Wedderburn, A., y Scholarios, D. (1993). Guidelines for shiftworkers: trials and errors?. Ergonomics, 36, 239-246. 